\title{
Circuit court du marché des produits agricoles: pour une gestion efficace du paysage ouvert, cas du bassin-versant de Maningory, Madagascar
}

Annick Ravakal, Bruno S. Ramamonjisoal, Harifidy Rakoto Ratsimbal, Aina N. A. Ratovoson'

\author{
Correspondence: \\ Annick Ravaka \\ École Supérieure des Sciences Agronomiques, \\ Département des Eaux et Forêts, \\ Université d'Antananarivo, BP 175, Antananarivo 101, Madagascar \\ Email: ravakaannick@gmail.com
}

\section{RÉSUMÉ}

Séparés par le rideau forestier du Parc National Zahamena, les paysans du côté Ouest du bassin versant du Maningory vivent en grande partie de la riziculture irriguée, alors que sur le versant Est, la culture itinérante sur brûlis ou tavy caractérise les pratiques agricoles des paysans. Ils sont connus pour les cultures pluviales de riz, d'arachide, de haricot et les cultures de rente de girofle, de café, et de letchi. Le bassin versant est constitué de la forêt naturelle de l'Aire Protégée, de la forêt de reboisement d'eucalyptus et des zones marécageuses autour du Lac Alaotra. Cet ensemble forme une mosaïque de paysage ouvert-défini dans cet article comme l'ensemble des zones herbeuses et arbustives, des zones de culture sur formations dégradées, ainsi que des espaces cultivables en bas-fond et en bas de pente. Le constat est que les surfaces des rizières cultivables deviennent de plus en plus restreintes, incitant les paysans à coloniser les tanety ou le paysage ouvert pour les pratiques agricoles. Cet article tente de comprendre le rôle du circuit court de commercialisation des produits agricoles dans le changement d'occupation de ce paysage. Deux approches ont été utilisées à cette fin : la modélisation d'accompagnement à travers un jeu de rôle, et des entretiens individuels. Les résultats démontrent que l'économie paysanne est surtout basée sur une économie de subsistance. La plupart de la production agricole est destinée à l'autosubsistance, incitant ainsi une faible résilience du paysage et suggère une forte vulnérabilité face à l'économie de marché. Le rôle du marché dans les stratégies d'occupation du sol reste encore allusif mais intrinsèquement lié aux prix des produits agricoles et à l'accessibilité des sites.

\section{ABSTRACT}

Separated by the Zahamena National park, smallholder farmers in the western part of the Maningory watershed largely rely on irrigated rice cropping, whereas those on the eastern front mainly rely on cash crops cultivated using slash and burn practices known as tavy in addition to rice cultivation. The watershed is made up of a mosaic of open landscapes, defined in this article as all grasslands, degraded cropland, and agricultural land. This mosaic is interspersed with the forests of the Zahamena National Park as well as those of eucalyptus reforestation, and Lake Alaotra's marshlands. As cultivable rice parcels become increasingly scarce, farmers are pushed to extend their farming into the tanety and open landscape. This study aims to gain a better understanding of the role of local agricultural product markets commercialization in landscape changes. Two approaches were implemented to this end, namely companion modelling using role-playing games and personal interviews. Results show that small farming economy is largely based on local subsistence. Most of the agricultural production is used for personal consumption, leading to a weak resilience of the landscape and suggesting high vulnerability towards the market. The role of the market on land use strategies remains allusive and intrinsically linked to the product prices, as well as to the ease of access to locations.

\section{INTRODUCTION}

Mondialement, deux personnes sur sept dépendent de l'agriculture pour leurs besoins de subsistance (IFAD 2011). Sous les tropiques, l'agriculture est principalement de type familial (Bowman et Haberle 2010). Les paysans sont considérés, à toute échelle, comme des producteurs. Ils dépendent étroitement d'une chaîne de valeur de commercialisation et de consommation. A l'instar des pays sous-développés, les pratiques agricoles à Madagascar dépendent des besoins des paysans (Kull 2000), mais également d'une rationalité locale qui les pousse à agir (Darré et al. 2004). Dans un contexte plus large, le secteur politique constitue un facteur transversal dans leurs prises de décision (Vågen 2006). L'agriculture est souvent pratiquée dans un paysage hétérogène et dynamique (Burel et Baudry 1999). II se traduit par un changement de l'environnement en fonction des objectifs individuels des paysans ainsi que des processus sociaux-économiques qui les entourent (Caillault et Marie 2009, Sayer et al. 2013). L'activité agri- 
cole, pratiquée dans un système socio-écologique complexe, considère la terre comme une unité paysagère. II est alors primordial de comprendre la perception de la réalité par les paysans et d'identifier les facteurs qui peuvent influencer de manière directe ou indirecte leurs stratégies d'occupation du sol (Godelier 1984, Deffontaines et Petit 1985).

La forte croissance démographique à Madagascar, avec un taux d'accroissement annuel de 2.8\% (Unfpa 2016), entraîne une insuffisance notable en terres cultivables. Les surfaces rizicoles sont souvent limitées aux vallées alluvionnaires, et les forêts sont soumises à des initiatives de conservation à l'encontre de la déforestation, laissant l'alternative de mise en valeur du paysage ouvert comme prometteuse. Entre les trois types d'écosystèmes globaux formés par les forêts, tanety, et zones humides ou marais qui coexistent dans le bassin-versant de Maningory, cet article traitera uniquement de I'utilisation des tanety. En s'inspirant de la classification de Moat et Smith (2007), le paysage ouvert, défini dans cet article sous le terme de tanety, est composé d'une mosaïque de zones herbeuses et arbustives, d'une forêt dégradée issue de la pratique agricole sur brûlis ou tavy, ainsi que d'une zone de culture en bas-fond et en bas de pente. Cet article essayera de mettre en valeur la potentialité de ces espaces ouverts, sachant qu'ils constituent près de $80 \%$ de la superficie totale de la zone d'étude. A l'échelle du bassin-versant, les produits agricoles sont limités aux produits de riz, de quelques cultures pluviales annuelles et de cultures de rente. Ces produits sont faiblement mis sur le marché, et souvent sur un circuit court de commercialisation (Bernard et al. 2007, Randrianarison et al. 2009).

L'hypothèse avancée par cet article est que les pratiques agricoles individuelles des paysans influencent la configuration du paysage sur le long terme. La principale question est de comprendre comment ce circuit de commercialisation des produits agricoles influence les stratégies d'occupation du paysage ouvert. Appréhender une meilleure gestion de cette ressource insinue une compréhension de sa structure et de son fonctionnement. Deux approches ont été couplées pour la collecte des données: la modélisation d'accompagnement à travers un jeu de rôle (Etienne 2014), et la méthode d'entretien individuel selon un questionnaire directif utilisé dans la science sociale.

\section{MATÉRIELS ET MÉTHODES}

PRÉSENTATION DE LA ZONE D'ÉTUDE. Le bassin versant de Maningory se situe sur la partie Est de Madagascar, à cheval entre les régions Alaotra-Mangoro et Analanjirofo (Wilmé et al. 2012). II est constitué d'une grande mosaïque de paysages à forêts denses humides $(13,46 \%)$, d'une formation secondarisée de forêts dégradées $(34,28 \%)$, de paysages herbeux $(36,58 \%)$, de zones de cultures $(9,32 \%)$ et de zones marécageuses $(4,20 \%)$, d'après une analyse de l'occupation du sol en 2014 (Randriamalala 2015). Le paysage ouvert concerne toutes les zones herbeuses et arbustives, cultivées ou non, les zones de culture sur les versants des collines et les formations de savoka — une jachère arborée et arbustive issue de la pratique de la culture sur brûlis. Quatre communes ou sites, représentants différentes variantes de paysages ouverts, ont été choisis d'amont en aval: Ambohijanahary, Vohimenakely, Antanandava et Vavatenina (Figure 1).

COLLECTES DES DONNÉES. Sur la partie occidentale du bassin versant, le mot tanety décrit la pente d'une colline et

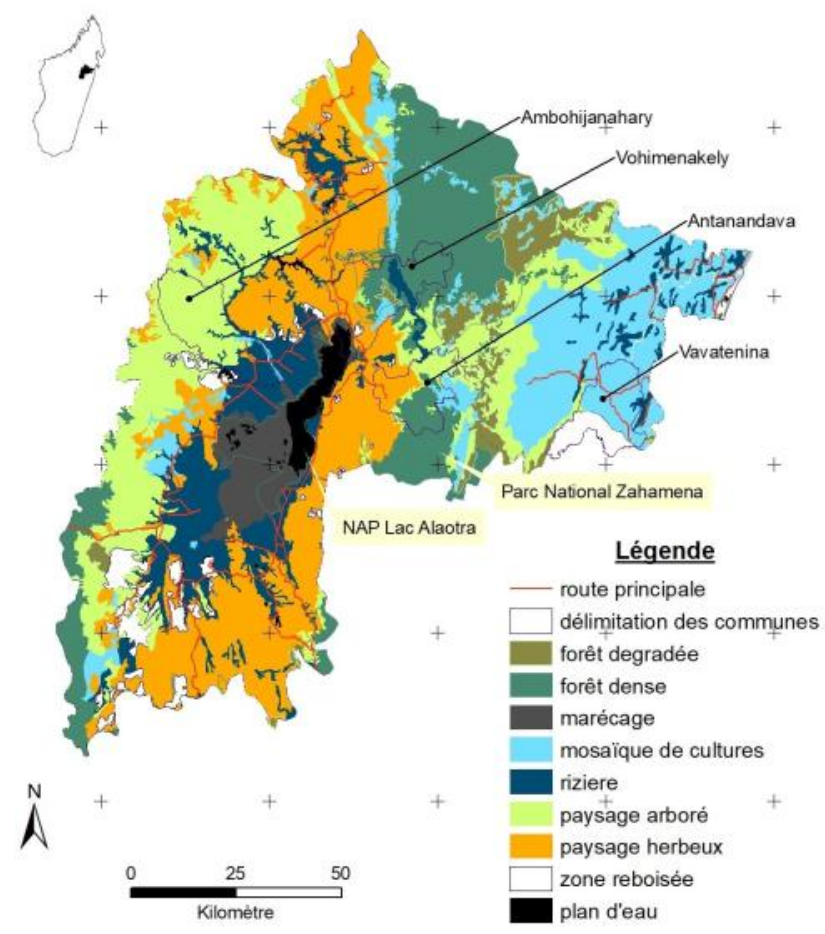

Figure 1. Localisation de la zone d'étude. Les quatre communes choisies pour la collecte des données formant un transect d'Ouest en Est du bassin-versant de Maningory.

la crête des montagnes, souvent colonisées par des herbacées telles que Aristida multicaulis et Imperata cylindrica. Le sol y est alors souvent lessivé et peu fertile. II est destiné aux pâturages, aux activités de reforestation et à la culture annuelle de quelques variétés pluviales d'arachide, de pois de terre ou de manioc. Quant à I'Est, il est composé par les versants de collines et de montagnes, souvent autour d'un lambeau de forêt naturelle. Dans son ensemble, le bassin versant de Maningory est majoritairement composé d'un paysage herbeux et arbustif avec quelques vestiges de forêts primaires. Par souci de concertation sur le terme utilisé, dans cet article tanety est défini comme un composant global du paysage du bassin-versant désignant les espaces ouverts, à l'exception des espaces boisés et des marais.

La structure et le fonctionnement du paysage est un système complexe formé de plusieurs composantes et d'acteurs locaux. cet article se focalise sur l'identification des facteurs pertinents pouvant influencer ce système et par conséquent l'occupation du sol, à travers les activités agricoles des paysans. Les entretiens semi-directifs effectués contribuent à comprendre la structure de ce système socio-écologique, avec une orientation sur l'accessibilité des sites par rapport aux routes, les superficies des espaces cultivés en riz irrigué, en d'autres cultures vivrières pluviales et en cultures de rente ; la production totale par individu enquêté et la répartition des récoltes pour l'autoconsommation des ménages et la vente. Outre les questions relatives aux pratiques agricoles et à leur écoulement sur un système d'autoconsommation ou de marché, les retombées des facteurs sociaux tels que la position familiale et l'origine sociale ont aussi été évoquées dans le questionnaire de collecte de données. Un entretien directif auprès de 326 individus répartis entre les quatre sites a alors été effectué afin de comprendre la structure de ce paysage (Table S1). L'objectif a été de définir si le circuit de commercialisation des produits conduit à un changement de pratiques agricoles et subséquemment à l'agencement du paysage global. 
Cette organisation des différentes composantes du paysage implique le besoin d'appréhender son fonctionnement. La modélisation d'accompagnement comprend des outils pour anticiper d'une manière participative cette tendance d'évolution du paysage. Parmi ces outils, les ateliers «AR(D)I » (Etienne et al. 2011) entrepris dans les sites montrent une description des acteurs concernés ainsi que leurs interactions avec les ressources attribuées dans I'utilisation du paysage ouvert (Figure 2). Un « jeu de rôle » sur le paysage ouvert a été conçu par la suite, afin de représenter de manière simple, implicite et ludique ledit paysage. Cette approche de modélisation d'accompagnement et de jeu de rôle présente la même structure de base que celle réalisée dans les marais autour du Lac Alaotra (cf. Reibelt et al. 2017). La dynamique de changement de couverture du sol ainsi que les modes agricoles d'utilisation des terres ont été décrits schématiquement à cet effet (Figure 3). Le jeu a été développé de manière participative grâce à la méthode de modélisation d'accompagnement (Barreteau et al. 2003). Vingt et une sessions ont été réalisées avec des paysans des quatre communes et de quelques représentants d'autorités locales. Une partie des résultats est utilisée dans cet article afin de comprendre le fonctionnement du paysage suivant la perception des joueurs. La table de jeu est une maquette de mosaïque de paysage composée de 18 parcelles. Les joueurs ont à leur disposition toutes les ressources reflétant les conditions réelles, nécessaires pour travailler individuellement leurs lopins de terre et subvenir à leurs besoins. Les facteurs pouvant modifier la trajectoire du paysage ont été traduits comme scénarios dans les tours de jeu. Le point commun des trois scénarios est l'intégration du rôle du marché et de la tenure foncière. Le premier scenario est considéré comme référence, suivi par l'introduction du facteur migration dans le deuxième scenario, et la tendance de la dégradation des forêts dans le troisième scenario. Dans chaque tour, les joueurs émettent leurs propres stratégies d'utilisation du sol pour subvenir à leurs besoins (Figure 4 et Figure 5). L'objectif est de comprendre, à travers leurs actions individuelles, la trajectoire du changement d'occupation du sol à l'échelle du paysage.

A l'instar des travaux de recherche publiés dans les journaux scientifiques à Madagascar et de par le monde, la réalisation de cet article a respecté les codes de conduite sur l'éthique (Wilmé et al. 2016). Les entretiens et les sessions de jeu ont été effectués de façon anonyme et confidentielle, priorisant ainsi le consentement de chaque individu.

\section{RÉSULTATS}

LA RIZICULTURE IRRIGUÉE : UNE TRADITION PLUTÔT QU'UNE ACTIVITÉ AGRICOLE. Le riz, une monoculture courante dans le bassin-versant de Maningory comme dans toutes les régions de Madagascar, accapare la plupart des terres arables, surtout les espaces irrigués. Le système agraire des paysans dépend, entre autres, de leur commune d'origine, de leur appartenance ethnique et de leur superficie agricole. Ces facteurs jouent un rôle prépondérant dans l'explication de la répartition des produits agricoles à l'autoconsommation des ménages et à la vente, mais également à la relation entre ces facteurs et l'accessibilité des gens aux infrastructures routières.

Les cultures sur tanety et sur bas-fond semblent toujours être interconnectées dans la stratégie des paysans (Table S2). La récolte des cultures vivrières pluviales offre un complément de source de revenu, alors que le riz est départagé entre l'autocon-

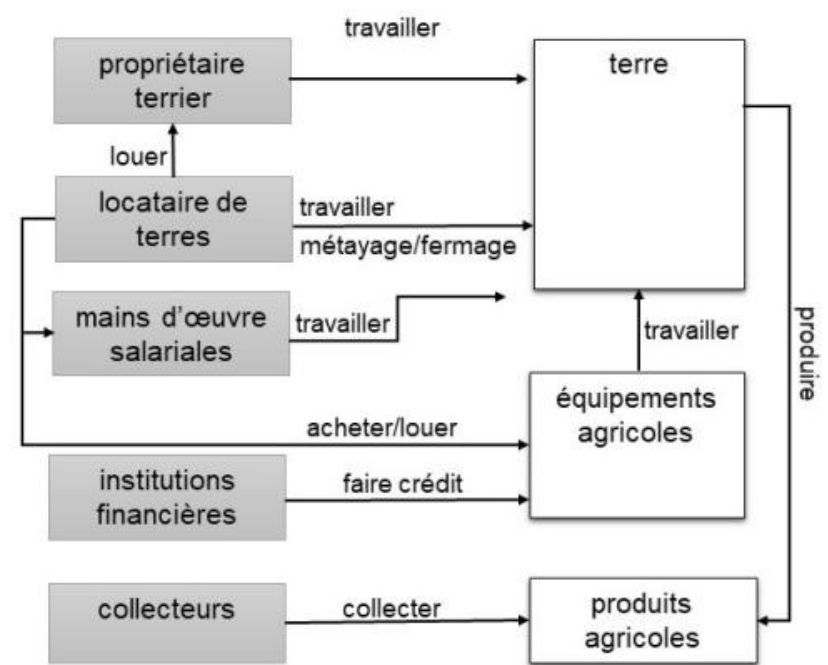

Figure 2. Modèle conceptuel montrant l'interaction entre les acteurs et les ressources, basé sur la méthode AR(D)I ou Acteurs, Ressources, Dynamiques et Interaction (Etienne et al. 2011)

sommation et la vente sur le marché régional et national. Les deux sous-bassins versants de Sahamaloto et d'Anony, dans la commune d'Ambohijanahary au nord du Lac Alaotra, alimentent quelques grandes exploitations familiales réparties sur 8000 ha de périmètre irrigué. Cette zone est composée d'une population de l'ethnie Sihanaka, des paysans habitant autour du lac et approvisionnant le " grenier à riz » de Madagascar. En 1946, lors du temps de la colonisation française, une politique publique de l'État a contribué à façonner cette localité avec une stratégie appelée « opération Lac Alaotra ». Depuis cette époque, la riziculture irriguée s'est améliorée grâce à la gestion de la fertilisation et à la maîtrise de l'eau à l'instar des aménagements hydro-agricoles. Elle s'est peu à peu développée grâce à une agriculture mécanisée, permettant de multiplier les rendements agricoles et passer de la subsistance à la commercialisation. Malgré que la zone soit considérée comme grande productrice de riz, les paysans attribuent une bonne part de leurs récoltes à l'autoconsommation des ménages. D'autant plus que l'étendue des superficies cultivables se trouve être de plus en plus restreintes à cause de la pression foncière, la croissance démographique et d'autres facteurs socio-économiques (Fujiki et al. 2015). L'agriculture reste alors, pour la plupart, de type familial et la production restante est subdivisée entre la semence pour la prochaine saison culturale et la vente à petite échelle. Cette politique de renforcement de la politique agricole s'est prolongée durant toute la première République à Madagascar (1960-1972) (Alain et al. 2007). A travers l'idéologie socialiste imposée par l'Etat à cette époque, toutes les initiatives agricoles se sont concentrées sur la production de riz même au dépit des communautés paysannes à cause d'une hausse considérable de l'importation. La mise en valeur des tanety a été laissée pour compte par manque de technicité et d'une assise stratégique stable.

Au Sud du Lac, dans les communes de Vohimenakely et d'Antanandava, les superficies rizicoles sont plus restreintes, de 1020 à 3000 ha. Partagés entre les ethnies Sihanaka et Betsimisaraka, les paysans se ruent de plus en plus vers les cultures sur tanety. Ce type de culture est vivement monté en puissance, particulièrement depuis 1945 (Penot et al. 2014), autour des forêts naturelles. Le seul vestige de forêt important existant dans le bassin versant est le Parc National de Zahamena, vivement menacé par la pratique de la culture itinérante sur brûlis ou 


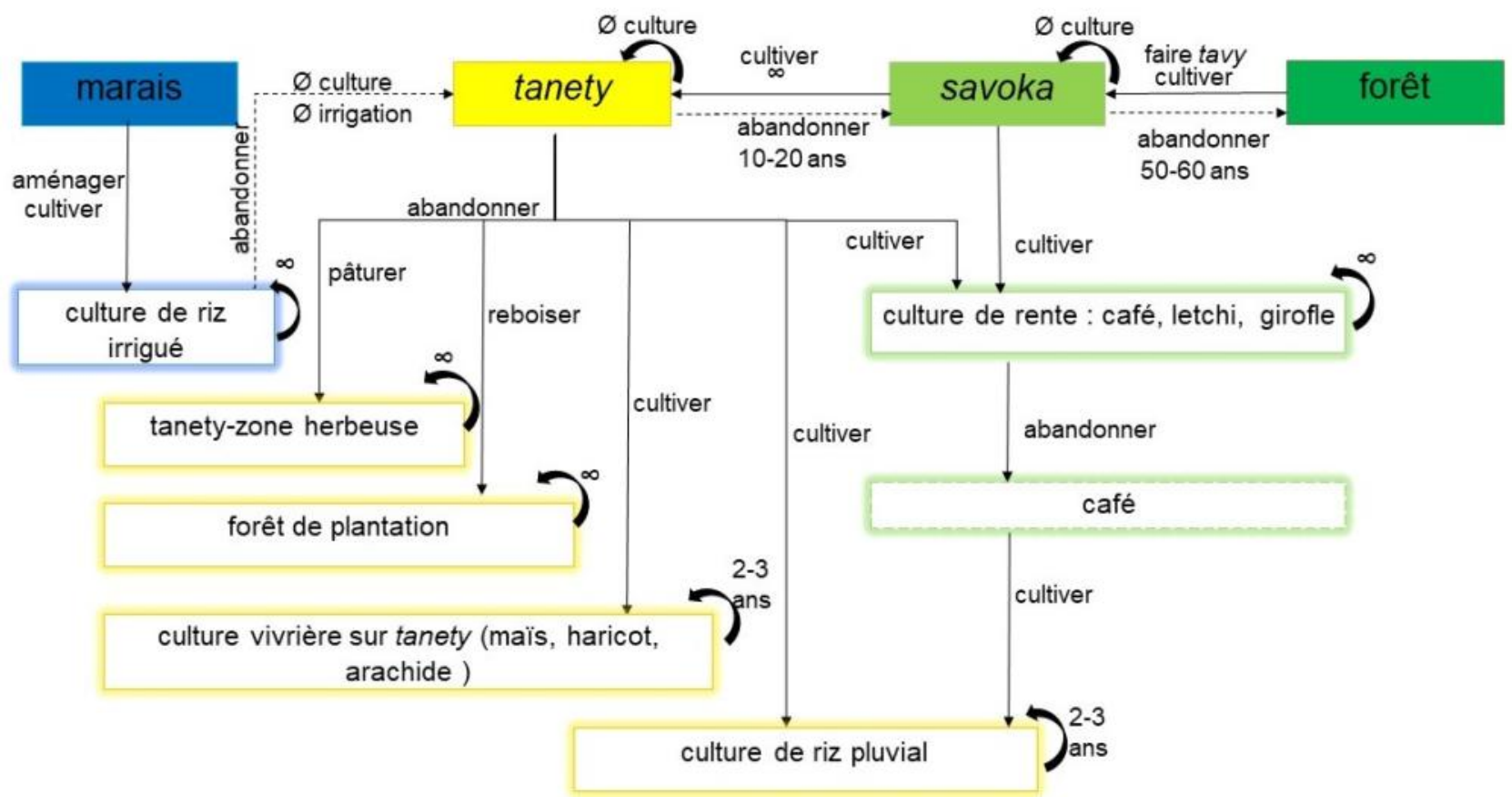

Figure 3. Diagramme de changement d'occupation et de couverture du sol à l'échelle du paysage. Les flèches en arc représentent une utilisation du sol sur des années consécutives, soit pour une durée de 2 à 3 ans, soit indéfiniment. Pour les périodes de reconstitution des tanety en savoka et des savoka en forêt, se conférer à Styger et al. 2006

\begin{tabular}{|c|c|c|}
\hline scenario 1 & scenario 2 & scenario 3 \\
\hline \multicolumn{2}{|c|}{ paysage initial LO(a) } & paysage initial $L O(b)$ \\
\hline \multicolumn{2}{|c|}{3 parcelles de forêt } & 18 parcelles de \\
\hline \multicolumn{3}{|c|}{6 parcelles de savoka } \\
\hline \multicolumn{3}{|c|}{7 parcelles de tanety } \\
\hline \multicolumn{3}{|c|}{2 parcelles de marais } \\
\hline \multicolumn{3}{|c|}{6 tours } \\
\hline \multicolumn{3}{|c|}{$\begin{array}{l}\text { augmentation de la charge à payer pour chaque tour à } \\
\text { partir du } 4 \text { e tour }\end{array}$} \\
\hline \multicolumn{3}{|c|}{$\begin{array}{l}\text { hausse du prix du riz au } 4 \mathrm{e} \\
\text { tour }\end{array}$} \\
\hline $\begin{array}{l}\text { joueurs tous natifs } \\
\text { de la commune }\end{array}$ & $\begin{array}{l}\text { joueurs natifs } \\
\text { et migrants } \\
\text { (répartis } \\
\text { aléatoirement) }\end{array}$ & $\begin{array}{c}\text { joueurs tous natifs } \\
\text { de la commune }\end{array}$ \\
\hline
\end{tabular}

Figure 4. Description des scénarios d'une session de jeu de rôle. En total, il y a trois scénarios, chacun composé de six tours, joués suivant les mêmes règles à l'exception du paysage de départ et des caractéristiques de joueurs. La table de jeu est composée de 18 parcelles réparties de façon similaire LO(a) pour les deux premiers scénarios et en $\mathrm{LO}(\mathrm{b})$ pour le 3e scénario.

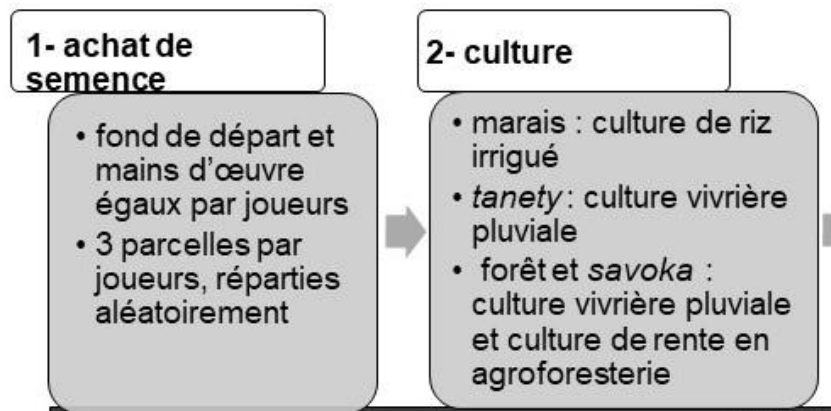

tavy. Les paysans étendent petit à petit leurs surfaces agricoles, situées autour de la forêt, pour subvenir à leurs besoins. Au cœur du bassin, les surfaces rizicoles disponibles sont plus limitées. Successivement, seuls 25 à $50 \%$ et 10 à $25 \%$ des ménages dans les communes de Vohimena et d'Antanandava pratiquent la riziculture sur zones irriguées. La production est donc principalement consacrée à l'autoconsommation.

La commune de Vavatenina, en aval du bassin-versant (Figure 1), est habitée par une population Betsimisaraka. Elle est connue pour une forte pratique du tavy, notamment pour le riz pluvial et les produits d'exportation tels que le girofle, le café et le letchi. La culture de rente, contrairement aux cultures annuelles pluviales du front Ouest, constitue la principale source de revenu pour les paysans. La riziculture irriguée est réalisée de manière secondaire dans cette région, avec seulement 25 à $50 \%$ de la population la pratiquant. Les paysans préfèrent vendre leur produit de riz, chaque kilo rapportant autour de 200 à 500 Ariary en 2015, et acheter du riz importé pour leur propre consommation. Les revenus issus du riz sont importants pour les paysans. Ils leurs permettent de subvenir à leurs besoins quotidiens de premières nécessités tels que l'achat de sucre, de gros sel, d'huile de cuisson et de pétrole pour les lampes d'éclairage. Accessoirement, le surplus est rajouté au budget pour payer les salaires des

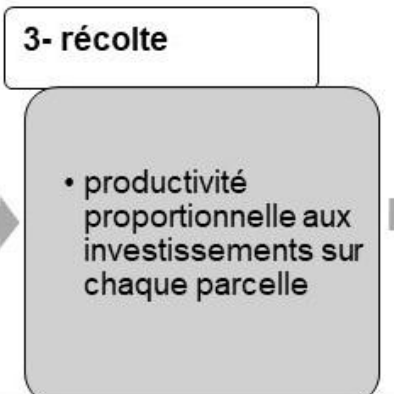

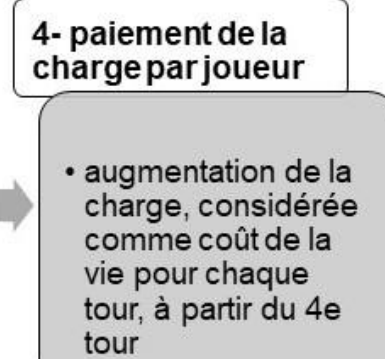

Figure 5. Description d'un tour de jeu de rôle. 
mains d'œuvre travaillant et cultivant les terres au début de la saison, coïncidant avec la période de soudure. Pendant cette période, les paysans combinent les pratiques agricoles à d'autres sources de revenus telles que la fabrication d'huile essentielle de girofle et les activités minières grâce à une migration temporaire.

Une analyse de régression multiple, au seuil de significativité de $5 \%$, montre que ni le prix du kilo du riz (valeur $p=0.445$ ) ni la distance du marché (valeur $p=0.655$ ) n'ont une incidence statistiquement significative sur la quantité de riz mise sur le marché par chaque ménage, et de ce fait sur la superficie cultivée. La riziculture est plutôt considérée comme partie intégrante de la coutume malgache et se perpétue de génération en génération. Malgré la faible plus-value octroyée par la culture de riz, les paysans lui accordent une attention particulière. Elle détient une place importante dans les habitudes alimentaires et même dans la tradition familiale. Dans le bassin-versant de Maningory, surtout en amont où les paysans disposent de grandes superficies rizicultivées, pouvoir subvenir aux besoins du ménage jusqu'à la prochaine récolte et avoir une possibilité d'alimenter directement les marchés régionaux et nationaux en riz constituent un indicateur de richesse. Malgré le fait qu'il n'existe pas de marché réellement formel, le prix du riz fluctue rarement, vendu entre 1200 et 1500 Ariary le kg selon les données d'enquête en 2015. Pour les communes au nord du Lac Alaotra et certaines communes productrices de riz à l'extrême Est du bassin versant, une partie des produits est accumulée par les collecteurs locaux. IIs les acheminent directement vers les grandes villes par leurs propres moyens. La part restante est recueillie par les collecteurs externes, qui viennent jusqu'aux chefs lieu des communes après la saison de récolte.

COLONISATION DES TANETY : VERS UNE MEILLEURE OCCUPATION DU PAYSAGE OUVERT. Les espaces ouverts sont très peu mis en valeur dans la zone d'étude. Alors que les superficies rizi-cultivées sont devenues plus restreintes à cause de la croissance démographique, les paysans se ruent peu à peu vers les cultures sur tanety. Globalement dans la zone d'étude, les cultures les plus pratiquées sont les cultures annuelles pluviales d'arachide et de haricot, ainsi que les cultures pérennes de produits de rente tels que le girofle et le letchi (Figure 6). L'espace ouvert décrit dans cet article couvre $80 \%$ de la superficie totale du bassin. Chaque ménage détient en moyenne 0.2 ha d'espace ouvert cultivé.

Uniquement $5 \%$ des paysans de la commune Ambohijanahary s'adonnent à la culture sur tanety. Sa valorisation est considérée comme un investissement non rentable, à cause de la faible fertilité du sol et d'une forte exposition à l'érosion. Au cœur de la zone d'étude, 10 à $50 \%$ des paysans des communes de Vohimena et d'Antanandava pratiquent la culture sur tanety. Ces espaces ont une faible accessibilité routière, une sécurité vulnérable rendant difficile et couteuse I'utilisation des équipements agricoles ainsi que le transport des récoltes. Dans la commune de vavatenina, plus de $80 \%$ de la population pratique le tavy. Cette habitude culturale est considérée comme une des causes directes de la dégradation des forêts sur le front oriental malgache et contribue à l'extension de la superficie des espaces ouverts.

Une analyse de régression linéaire multiple a permis de comprendre que la quantité de produits sur tanety augmente en fonction du prix sur le marché (valeur $p<0.001$ ), et entraîne une augmentation de la superficie cultivée. Les résultats des entretiens auprès des paysans montrent qu'une grande partie de la production est écoulée sur le marché local. Toutefois, certains types de produits, tel que le haricot et l'arachide arrivent à approvisionner les marchés des grandes villes de Toamasina et même de la capitale Antananarivo. En effet, ces villes sont reliées par les principales routes nationales existantes dans le bassin-versant. Elles sont constituées par la Route Nationale 22 de Vavatenina jusqu'à la bifurcation, qui la relie à la Route Nationale 5 conduisant à Toamasina où se situe le premier port de Madagascar, puis donnant vers le sud sur la Route Nationale 2 menant à Antananarivo. La Route Nationale 44 est additionnée à cette liste, quoique plus ou moins délaissée compte tenu de son mauvais

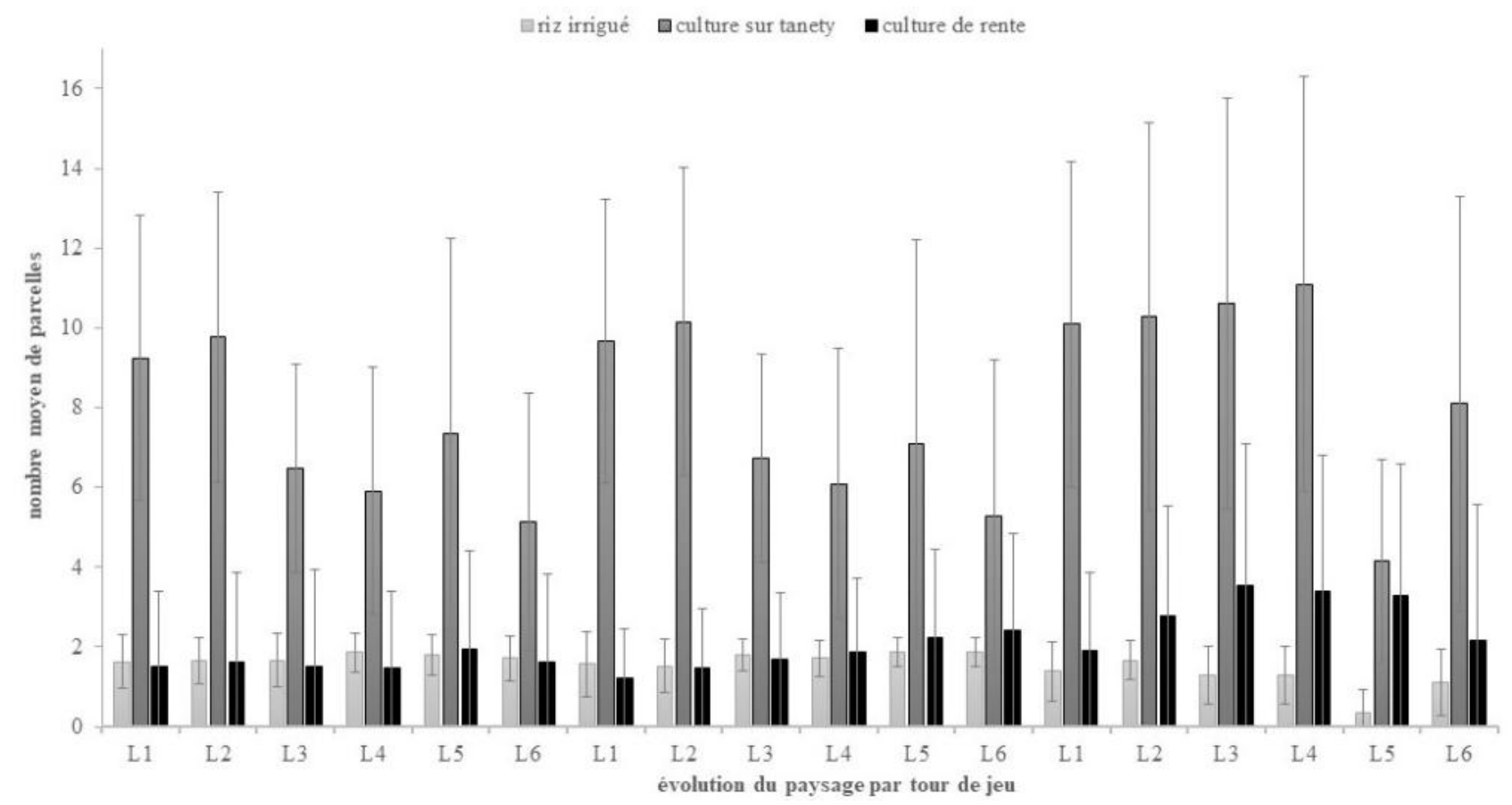

Figure 6. Répartition des modes d'utilisation du sol sur 21 sessions de jeu ; les barres d'erreur représentent les écart-types. 
état, surtout durant la saison de pluie. Elle sert ainsi au transport des produits sur tanety à l'échelle régionale et de riz irrigué sur le marché national.

La commercialisation des produits de rente est considérée comme une source de revenu d'appoint pour les paysans. Une fois que les plantes pérennes ont fructifiées, elles produisent d'une manière conséquente chaque année, à l'exception du girofle. La récolte de clous de girofle reste aléatoire jusqu'à I'ouverture de la campagne de collecte et offre un revenu sporadique, alors que les feuilles sont utilisables toute l'année pour produire de l'essence. Sur le marché, au niveau des collecteurs locaux, l'essence de girofle est vendue à 30000 Ariary par litre. Les paysans produisent alors de l'essence presque toute I'année afin de répondre à leurs besoins financiers. Les années où les récoltes de clous sont bonnes, des gens jouant à la fois le rôle de collecteur et de docker auprès des paysans collectent les clous de girofle préparés, à raison de 18000 Ariary le kg, les transportant sur leur dos à l'aide de deux sacs fixés de part et d'autre d'une tige d'arbre ou de bambou. Ils les vendent par la suite aux collecteurs locaux se situant dans les chefs-lieux de la commune à raison de 22000 Ariary le $\mathrm{kg}$. Ces derniers revendent les produits aux grandes sociétés d'exportation à Toamasina et à Antananarivo.

Contrairement à la récolte de clou de girofle, les fruits du letchi sont plus stables et bénéficient d'un autre circuit de commercialisation. Ils représentent ainsi une source de revenu plus ponctuelle pour les paysans. Au moment de l'ouverture de la campagne de récolte, les collecteurs externes viennent avec des camions et des fourgonnettes pour collecter les paniers de letchis de 20 à $25 \mathrm{~kg}$, déjà préparés par les salariés des propriétaires des pieds de letchis. Ces produits sont déposés tout au long de la Route Nationale $n^{\circ} 22$ et sont vendus pour 600 à 750 Ariary le kg.

Enfin, la culture de la vanille a été abandonnée par les paysans de cette commune depuis une dizaine d'année à cause d'une baisse de prix et d'une insécurité accablante. Les paysans qui ont travaillé dans la production de vanille ont alors migré vers d'autres communes au Nord de la région Analanjirofo. La production de café a subi le même sort. La chute du prix depuis une dizaine d'années a poussé les paysans à convertir leurs champs de caféiers en cultures de riz pluvial. Le reliquat de production de café n'est mis sur le marché que si le cours est attractif ou en cas de besoin d'argent pressant, ce qui n'est que dérisoire.

ACCESSIBILITÉ, MIGRATION ET TENURE FONCIÈRE.

L'accessibilité aux routes facilite le transport des produits. Les paysans des communes disposant d'une bonne accessibilité ont une plus grande superficie cultivée et une plus grande quantité de production pour la vente. En comparant les données de simulation de l'évolution des parcelles occupées par la riziculture pour l'ensemble des sessions de jeu de rôle, on observe une augmentation de $33.5 \%$ entre le scenario 1 et le scenario 2 avec les facteurs "marché » et "migration ». Cette proportion est de 94.2\% entre le scenario 1 et 3 en ajoutant les facteurs « dégradation du sol » et " tenure foncière ». A l'instar de la réalité, les rizières irriguées sont limitées par les bas-fonds et les vallées alluvionnaires dont les superficies restent immuables. Ainsi, les paysans ayant une faible accessibilité aux routes ont aussi un accès limité au marché. Ils attribuent alors une grande part de leur production en riz à la consommation de leur ménage.
Une migration de courte distance est souvent observée sur le front Ouest du bassin où les jeunes natifs ayant travaillé en tant que salarié dans d'autres communes reviennent dans leur commune natale pour hériter des terres de leurs parents ou pour acheter d'autres terres agricoles. Les propriétaires terriens sont des natifs de la localité, surtout concernant les rizières irriguées. Par contre, les migrants de longue distance, issus des ethnies Merina et Betsileo, travaillant en tant que main d'œuvre salariale, se sont peu à peu installés dans la commune. A l'ouest du bassin versant, les migrants occupent les tanety pour les cultures annuelles pluviales. Alors que sur le côté Est, où les tanety résultent souvent de la pratique de tavy sur les savoka, ils appartiennent aux gens natifs de la région qui ont pratiqué les cultures sur brûlis successives sur la forêt ou ont hérité de leurs parents pour faire place, dans le long terme, aux cultures de rente de girofle, de letchi et de café.

Ce résultat est renforcé par l'analyse de régression qui a démontré une corrélation significative entre l'origine des paysans en tant que migrant ou natif et la quantité de produits de rente qu'ils mettent sur le marché (Table S2). Le fait d'être natif de la commune augmente sensiblement l'accessibilité au marché, et donc également la quantité mise en vente. Quoi qu'il en soit, la migration ouvrière de longue distance sur le versant Est n'est que temporaire. Les gens sont attirés par le marché d'emploi, créé par la collecte et le conditionnement des produits de rente, les services de location de voiture pour le transport des produits ou la pratique de la bijouterie artisanale grâce à l'épanouissement de I'orpaillage aux alentours du Parc National pour les hommes et le commerce de vêtements ou d'autres produits artisanaux pour les femmes. L'accessibilité aux routes nationales accentue ce phénomène de migration. Une corrélation évidente entre la quantité agricole produite et l'accessibilité aux routes a été définie (Table S2). L'existence de ce courant migratoire renforce la conjoncture économique des produits agricoles et incite à une augmentation de la quantité de produits mise en vente par les paysans. Toutefois, les paysans font face à d'autres problèmes économiques, comme l'irrégularité des prix des produits sur le marché. Ils sont souvent fixés par les collecteurs et entraînent un impact sur l'écoulement des produits et sur les superficies cultivées.

\section{DISCUSSION}

La pratique de la riziculture irriguée tend plus vers une économie de subsistance qu'à une économie de marché, à l'exception de quelques grandes exploitations dans les périmètres irriguées du Lac Alaotra et certaines communes disposant de plaines irrigables sur la côte Est du bassin-versant. Ce type de culture est plutôt considérée comme une coutume avant d'être une activité agricole, le savoir-faire agricole est transmis de génération en génération (Cholez et al. 2010). Les paysans autochtones ont, pour la plupart, hérités des terres de leurs parents (ibid). Les rizières irriguées cultivables sont souvent limitées aux vallées alluvionnaires. Par conséquent, l'augmentation de la population a favorisé l'exploitation des tanety à des fins agricoles. Un paysan sans terre migre physiquement et économiquement pour survivre, en poursuivant des activités agricoles. Sur le versant Ouest du bassinversant, les migrants occupent plus les tanety que les autochtones. Toutefois, une étude effectuée par Bertrand et Lemalade en 2003 dans la commune de Didy a démontré qu'ils 
viennent à se concurrencer avec les natifs faute de marais aménageable en rizière irriguée.

L'utilisation des tanety a pris plus d'envergure depuis une trentaine d'années (Durand et Nave 2008) surtout pour la recherche de cultures de rente prometteuses. Cette vision d'introduire l'économie de marché dans les sciences sociales ou « ethnographie économique » est apparue entre les années 1970 et 1990 (Dufy et Weber 2007). II s'agit de décrire une assise naturelle au mécanisme de marché et à l'économie de subsistance par une « théorie néoclassique » complexe dite de "Polanyi » (ibid).

Les paysans du front Ouest utilisent les tanety pour les cultures annuelles pluviales. Sur l'Est, ces espaces sont issus de la pratique de tavy sur des formations secondarisées, pour faire place successivement à une culture de riz pluvial, puis à d'autres cultures vivrières pour se terminer avec des cultures pérennes, de rente, jouant souvent un rôle important en tant que marqueur foncier (Rabemananjara 2014). Dans cet article, les cultures de rente sont souvent des cultures pérennes. Une précision entre ces deux types de cultures a déjà été évoquée en insistant sur le fait que la culture de rente est liée à une question d'accessibilité alors que les cultures pérennes sont guidées par une stratégie d'occupation foncière par les paysans (Bertrand et Le Roy 1991, Bertrand et Lemalade 2003). D'ailleurs, cette question foncière demeure encore un sujet de confusion à Madagascar (Bellemare 2009, Penot 2010)

La population rurale envisage toujours des revenus monétaires même dans les zones à fort enclavement (Bertrand et Lemalade 2003). Le terme de circuit de commercialisation évoqué dans cet article mentionne un parcours habituel entrepris par les paysans. II est relié à la vente de produits agricoles sur le marché Le marché affecte également la migration, qui est considérée à la fois comme une stratégie économique et sociale par les paysans (Deschamps et al. 1959, Andriamanalina et al. 2014). Le phénomène de migration agricole d'Ouest en Est du bassinversant de Maningory est particulièrement accentué par la commercialisation de produits de rente. En effet, la quantité produite augmente avec la hausse du prix sur le marché national. Cette situation entraîne implicitement une augmentation de la surface cultivée, induisant non seulement une extension des espaces ouverts et une augmentation du flux de migrants, mais aussi une dégradation de la couverture forestière (Rabemananjara 2014). L'espace ouvert occupe une grande proportion du paysage. Cette situation devrait être utilisée à priori, pour une amélioration de l'économie de subsistance vers une économie de marché.

L'approche, en termes de gestion des bassins versant, est encore un concept récent. Malgré que la question de migration interne ou externe ait été évoquée vers les années 1960 (Durand et Nave 2008), elle reste peu documentée et encore non articulée dans la politique de l'Etat. Les prochaines actions devront alors être axées sur l'articulation tanety / forêts / zones humides à travers une régularisation des marchés agricoles surtout pour la stabilisation des prix des produits à l'exportation. Malgré les savoir-faire acquis pour la mise en valeur des tanety depuis les années 1980 (Penot 2010), la récolte des cultures annuelles pluviales reste encore dérisoire pour un circuit de commercialisation à grande échelle. Un appui pour l'adoption des systèmes d'innovation tels que l'agriculture de conservation (Penot 2010, Andriarimalala et al. 2013) mérite encore la préoccupation des acteurs de développement. La valorisation des tanety implique une prise de risque par les paysans, basée sur une stratégie pour une amélioration de leur condition de vie (Brown et Everard 2015) et inconsciemment pour une gestion du paysage global.

Le jeu de rôle, dans cette étude, a été utilisé comme outil de modélisation d'accompagnement (Etienne 2014, voir également Waeber et al. 2017 pour le cas de l'Alaotra), et a permis de comprendre les facteurs pertinents qui ont pu influencer l'occupation du sol dans une mosaïque de paysage. II a facilité l'analyse de l'interaction entre les paysans et le système socio-écologique complexe qui les entoure en contribuant au développement du circuit de commercialisation des produits agricoles à une échelle locale. Cholez et al. (2010) décrivent ces paysans de Base of the Pyramid ou BoP, visant à instaurer un système de marché qui favorise les plus pauvres, tel le cas des paysans du bassin-versant. Le paysage ouvert gagnera indéniablement en étendue au détriment des zones boisées. À l'issu des jeux de rôle, les cultures pratiquées sur le paysage ouvert, qu'elles soient vivrières ou de rente, gagneront largement de l'ampleur au cours du temps, compte tenu des facteurs économiques et sociaux qui les conditionnent.

\section{CONCLUSION}

La colonisation des tanety dépend de plusieurs facteurs tels que les superficies de rizières cultivables disponibles, la fertilité du sol et le marché des produits agricoles. Toutefois, elle nécessite encore un développement agro-écologique et d'une assise politique conséquents, quant à son application. Les perceptions et les actions des paysans sont étroitement liées au contexte de commercialisation. Elles dépendent principalement d'une analyse de la résilience du marché de produits agricoles, en tenant compte de la dynamique sociale existante et de la diversité du paysage. L'utilisation conjuguée de l'approche de modélisation d'accompagnement et de la méthode d'entretien individuel en science sociale permet d'émettre une suggestion sur la gestion du paysage ouvert en se concertant sur les prochaines décisions. À travers un système paysans-exploitation-paysage, réside une dimension de perception sociale étroitement liée au contexte de production. Quoi que basée sur une économie de subsistance, l'économie paysanne cherche à faire jouer ensemble le concept de réciprocité et la force des stratégies individuelles au niveau des ménages. Toutefois, le rôle du marché reste encore allusif quant aux stratégies d'occupation du sol dans le paysage ouvert, par contre il est intrinsèquement lié à d'autres facteurs sociaux tels que l'accessibilité aux routes, la migration et le foncier. D'autres études, telles que la construction des modèles de changement d'occupation du sol, l'analyse des politiques publiques sur les questions foncières et de migration, permettront d'anticiper, de manière participative et concertée, les enjeux de la valorisation du paysage ouvert et contribuer à la mise en place d'une stratégie de gestion effective dans le long terme.

\section{REMERCIEMENTS}

Nous tenons à adresser nos sincères remerciements à l'égard des personnes suivantes : Claude Garcia (ETH Zurich), Lucienne Wilmé (Missouri Botanical Garden Madagascar), Patrick Ranjatson (ESSAForêts), et Patrick Waeber (ETH Zurich) pour leur appui dans la réalisation de cette recherche, particulièrement durant la phase de conception du jeu de rôle. Nous tenons également à exprimer toutes nos reconnaissances aux éditeurs et aux reviewers, pour leurs précieuses contributions à toutes les étapes d'améliorations et de publication de cet article. Nous tenons aussi témoigner notre gratitude à nos assistants de recherche: Rakotomalala 
Aimée Gisèle Vanessa, Randriamiharisoa Nirina Claudia, Randriamanjakahasina Olivier Pascal et Rakotoarivony Miotisoa Katuscia pour leur aide précieux durant la collecte des donnée sur le terrain. Enfin, cette recherche n'aurait pu aboutir sans un soutien financier. Nos remerciements s'étendent ainsi au Swiss Program for Research on Global Issues for Development, qui nous a octroyé une bourse de recherche à travers le projet AlaReLa - Alaotra Resilience Landscape. IZ01Z0_146852.

\section{RÉFÉRENCES}

Alain, P. B, Rivo, R. A., Lalaina, R. A. et Lydia, R. H. 2007. Les implications structurelles de la libéralisation sur l'agriculture et le développement rural. Available at <https://goo.gl/f18bia>

Andriamanalina, B. S., Burnod, P., Rakotomalala, H. \& Deschênes, S. 2014. Rural youth, agriculture and access land: The case of Madagascar. In: Conference on Land Policy in Africa. The Next Decade of Land Policy in Africa: Ensuring Agricultural Development and Inclusive Growth. pp 1-16. 11-14 November 2014, Addis Ababa, Ethiopia.

Andriarimalala, J. H., Rakotozandriny, J. N., Andriamandroso, A. L. H., Penot, E., Naudin, K., et al. 2013. Creating synergies between conservation agriculture and cattle production in crop-livestock farms: a study case in the Lake Alaotra Region of Madagascar. Experimental Agriculture 49, 3: 352-365. <http://dx.doi.org/10.1017/S0014479713000112>

Barreteau, O., Antona, M., D'Aquino, P., Aubert, S., Boissau, S., et al. 2003. Our companion modelling approach. Journal of Artificial Societies and Socia Simulation 6, 1. Availble at <http://jasss.soc.surrey.ac.uk/6/2/1.html>

Bellemare, M. F. 2009. Sharecropping, insecure land rights and land titling policies: A case study of Lac Alaotra, Madagacar. Development Policy Review 27 1: 87-106. <http://dx.doi.org/10.1111/j.1467-7679.2009.00437.x>

Bertrand A. et Lemalade J-L. 2003. Accès au marché, accès au foncier et migrations. In : Déforestation et Systèmes Agraires à Madagascar : Les Dynamiques des Tavy sur la Côte Orientale (Deforestation and Agrarian Systems in Madagascar: Tavy Dynamics on the East Coast). S. Aubert, S. Razafiarison et A. Bertrand (eds.), pp 167-175. CIRAD, Montpellier, FR.

Bertrand, R. \& Le Roy, E. 1991. Appui Méthodologique aux Volets "Foncier" et "Economie Forestière" (Première Mission). Programme "Hautes Terres". Opération de Recherche: la Production Forestière dans I"Économie Rurale sur les Hautes Terres Malgaches: Foncier, Ménages et Collectivités Rurales dans les Régions de Manjakandriana et de Vinaninony. Nogent-sur-Marne : CIRAD-CTFT, Montpellier, FR.

Bernard, P. B., Ramboarison, R., Randrianarison, L. et Rondro-Harisoa, L. 2007. Les implications structurelles de la libéralisation sur l'agriculture et le développement rural. Synthèse nationale, EPP/PADR - Ministère de l'Agriculture, de I'Elevage et de la Pêche/UPDR. APB Consulting. Available at $<$ https://goo.gl/WCTE6f>

Bowman, D. M. J. S. \& Haberle, S. G. 2010. Paradise burnt: How colonizing humans transform landscapes with fire. PNAS 107, 50: 21234-21235. <http://dx.doi.org/10.1073/pnas.1016393108>

Brown, I. \& Everard, M. 2015. A working typology of response options to manage environmental change and their scope for complementary using an ecosystem approach. Environmental Science \& Policy 52: 61-73. <http://dx.doi.org/10.1016/j.envsci.2015.05.006>

Burel, F. et Baudry, J.* 1999. Ecologie du paysage. Concepts, méthodes et applications. Annales de Géographie 110, $618: 201$.

Caillault, S. et Marie, M. 2009. Pratiques agricoles, perceptions et représentations du paysage : quelles articulations? Approches croisées Nord/ Sud. Norois 213, 4 : 9-20. <http://dx.doi.org/10.4000/norois.2995>

Cholez, C., Trompette, P., Vinck D. et Reverdy, T. 2010. L'exploration des marchés BoP : une entreprise morale. Revue Française de Gestion. 208-209.

Darré, J.-P., Mathieu, A. et Lasseur, J. (eds.). 2004. Le Sens des Pratiques. Conceptions d'Agriculteurs et Modèles d'Agronomes. INRA, Paris.

Deffontaines J. P. et Petit, M. 1985. Comment étudier les exploitations agricoles d'une région: présentation d'un ensemble méthodologique. INRA, Dijon, FR.

Deschamps, H.1959. Les migrations intérieures passées et présentes à Madagascar. Éditions Berger-Levrault, Paris. Available at <https://goo.g//icFjMt>
Dufy, C. et Weber, F. 2007. L'Ethnographie Economique. Editions La Découverte, Paris.

Durand, C. et Nave, S. 2008. Les Paysans de I'Alaotra, Entre Rizières et Tanety. Etude des Dynamiques Agraires et des Stratégies Paysannes Dans un Contexte de Pression Foncière, Lac Alaotra, Madagascar. Document de travail BV lac n¹0. Available at <https://goo.gl/1Adij9>

Etienne, M. (ed.) 2014. Companion Modelling. A participatory Approach to Support Sustainable Development. Éditions Quæ, Versailles, FR.

Etienne M., Du Toit, R. D. \& Pollard, S. 2011. ARDI: a co-construction method for participatory modeling in natural resources management. Ecology and Society 16, 1: 44. Available at <http://www.ecologyandsociety.org/vol16/iss1/art44/>

Fujiki, K., Mietton, M., Andriamasinoro, A., \& Andriamasinoro, W. 2015. The evolution of a rural territory at plot scale: Between hyper-fragmentation and land grabbing (irrigation perimeter PC15-Marianina Valley, Alaotra-Mangoro, Madagascar). Land Use Policy 43: 170-185. <http://dx.doi.org/10.1016/j.landusepol.2014.11.009>

Godelier, M. 1984. L'Idéel et le Matériel: Pensée, Économies, Sociétés. Fayard, Paris.

IFAD. 2011. Viewpoint: Smallholders can feed the word. Available at $<$ https://goo.gl/mNq1xd>

Kull, C. A. 2000. Deforestation, erosion, and fire: degradation myths in the environmental history of Madagascar. Environment and History 6, 4 : 423-450. $<$ http://dx.doi.org/10.3197/096734000129342361>

Moat, J., \& Smith, P. P. 2007. Atlas of the Vegetation of Madagascar. Royal Botanic Gardens, Kew.

Penot, E. 2010. Savoirs, pratiques et changement de paradigme: de l'agriculture irriguée à la colonisation des "tanety" (collines). Mythe, espoirs et réalités pour un développement durable au Lac Alaotra. . Cirad-Inra-SupAgro, Montpellier, FR. Available at <http://hal.cirad.fr/cirad-00768348/document>

Penot, É., Dabat, M.-H., Rakotoarimanana, A. \& Grandjean, P. 2014. L'évolution des pratiques agricoles au lac Alaotra à Madagascar. Une approche par les temporalités. Biotechnologie, Agronomie, Société et Environnement 18, 3 : 329-338.

Rabemananjara, Z. H. 2014 Migration causing forest degradation in Madagascar: prevention or adaptation to effects? Pinacle Natural Resources and Conservation. 1, $1: 194-201$.

Randriamalala, I. H. * 2015. Analyse de la Dynamique Spatio-Temporelle du Lac Alaotra et de I'Occupation du Sol dans le Bassin-Versant de Maningory. Unpub. M.Sc. thesis. Ecole Supérieure des Sciences Agronomiques, Université d'Antananarivo, Madagascar.

Randrianarison, L., Andrianirina, N. \& Ramboarison, R. 2009. Dimensions Structurelles de la Libéralisation pour l'Agriculture et le Développement Rural. Phase II. EPP/PADR Ministère de l'Agriculture, de l'Elevage et de la Pêche/UPDR and APB Consulting. Country case study carried out for RuralStruc Programme, Phase II Antananarivo, Madagascar. Available at <https://goo.gl/NJoiRz>

Reibelt, L. M., Moser, G., Dray, A., Randriamalala, I. H., Chamagne, J., et al. 2017 (In press). Tool development to understand rural resource users' land use and impacts on land type changes in Madagascar. Madagascar Conservation \& Development. <http://dx.doi.org/10.4314/mcd.wetlands.3>

Sayer, J., Sunderland, T., Ghazoul, J., Pfund, J.-L., Sheil, et al. 2013. Ten principles for a landscape approach to reconciling agriculture, conservation, and other competing land uses. Proceedings of the National Academy of Sciences of the United States of America 110, 21: 8349-8356. $<$ http://dx.doi.org/10.1073/pnas.1210595110>

Styger, E., Rakotondramasy, H. M., Pfeffer, M. J., Fernandes, E. C. M. \& Bates, D. M. 2006. Influence of slash-and-burn farming practices on fallow succession and land degradation in the rainforest region of Madagascar. Agriculture, Ecosystems \& Environment 119, 3-4: 257-269. $<$ http://dx.doi.org/10.1016/j.agee.2006.07.012>

Unfpa (United Nations Population Fund). * 2016. Population matters.

Vågen, T.-G. 2006. Remote sensing of complex land use change trajectories-a case study from the highlands of Madagascar. Agriculture, Ecosystems and Environment 115, 1-4 : 219-228. <http://dx.doi.org/10.1016/j.agee.2006.01.007> 
Wilmé, L., Ravokatra, M., Dolch, R., Schuurman, D., Mathieu, E., et al. 2012. Toponyms for centers of endemism in Madagascar. Madagascar Conservation \& Development 7, 1: 30-40. <http://dx.doi.org/10.4314/mcd.v7i1.6>

Waeber, P. O., De Grave, A., Wilmé, L. \& Garcia, C. A. 2017 (In press). Play, learn, explore: grasping complexity through gaming and photography. Madagascar Conservation \& Development. <http://dx.doi.org/10.4314/mcd.wetlands.1>

Wilmé, L., Waeber, P. O., Moutou, F., Gardner, C. J., Razafindratsima, O., et al. 2016. A proposal for ethical research conduct in Madagascar. Madagascar Conservation \& Development 11, 1: 36-39. $<$ http://dx.doi.org/10.4314/mcd.v11i1.8>

* Please contact the authors for the unpublished documents.

\section{SUPPLEMENTARY MATERIAL.}

Available online only.

Tableau S1: Liste thématique des variables utilisées dans le questionnaire de collecte de données.

Tableau S2: Les variables retenues et leurs valeurs $p$. 